\title{
Matched Filter Bounds on $q$-ary QAM Symbol Error Probability for Diversity Receptions and Multipath Fading ISI Channels
}

\author{
Heung-No Lee ${ }^{1}$ \\ Information Science Laboratory, \\ HRL Research Laboratories, L.L.C., BLDG 254, \\ 3011 Malibu Canyon Road, \\ Malibu, CA 90265 \\ Phone: (310) 317-5929, \\ FAX: (310) 317-5695 \\ heungno@hrl.com
}

\author{
Gregory J. Pottie \\ Electrical Engineering Department, \\ University of California at Los Angeles \\ P.O. Box 951594 \\ Los Angeles, CA 90095 \\ Email: pottie@icsl.ucla.edu \\ Phone: (310) 825-8150, \\ FAX: (310) 206-8495 \\ pottie@icsl.ucla.edu
}

Abstract - This paper extends previous works in matched filter bounds [2][3][4] and provides a theoretical calculation of detection probability of $q$-ary QAM signals averaged over the diversity reception and multipath fading ISI channels. The matched filter bounds are the best attainable detection performance at a given SNR, which may or may not be obtainable in practice. While an exact analytical expression of detection performance associated with a practical transceiver over multipath ISI channels is difficult to obtain, the matched filter bounds provide many useful information to be compared with the simulation results.

Technical Kewords: Matched filter bound, modulation, equalization and diversity combining.

1. Corresponding author 


\section{INTRODUCTION}

Based on the matched filter theory (see Chapter 6 of [1]), the detection SNR of any linearly filtered receive-signal is maximized if the matched filter, which can be obtained assuming the receiver has the perfect knowledge of the filter, is applied to the received signal perturbed by the additive noise. In this paper, we use the matched filters to derive the symbol error probability of $q$ ary QAM signals transmitted over the diversity reception and multipath fading ISI channels; for description of the communication channel, see Figure -1. A complete system of a transceiver involves many different function blocks, including a channel estimation and tracking, a synchronization, an adaptive diversity combining and equalization, and possibly an adaptive sequence decision [8][9]. In such systems, an exact calculation of detection probability is difficult and the approach may also dependent upon a particular selection of fading rate, detection and estimation conditions. Thus, most of times it is considered to be better off to perform simulation to obtain the exact detection performance of a system. Meanwhile, as a tool to calculate link-budget requirement or as a tool to support/verify the simulation results, a fundamental capacity calculation which can be computed relatively easily and applied regardless of any particular estimation and detection scheme is extremely useful.

Previous works in calculating the matched filter bounds on fading channels include Mazo [2], Clark et. al. [3], Ling [4], Proakis [6] and Jake [7]. In Jake and Proakis, the matched filter bounds are derived for the maximal ratio combining receiver, where each of the receive diversity-antennas in Figure - 1 is assumed to be a single tap Rayleigh fading. Mazo, Clark and Ling extended the results for multipath fading frequency-selective channels with BPSK, 4-PSK or QPSK signaling. Summarizing their approaches, we note that there are three major steps. The first is to obtain the bit error or symbol error probability (SEP) expression of a single and static channel. The second is to taking the expectation of the SEP over ensemble of the channel. This results in a well-known integral form which involves an integral of error probability function over a gamma distribution. The gamma distribution represents the probability density function, denoting the probability of matched filter SNR taking a particular value. The third part is to generalize the second to diversity reception and fading ISI channels. This part involves the use of eigenvalue decomposition to decorrelate the matched filter SNR. The matched filter SNR is the quadratic combination of correlated random variables, resulting from common transmit-shape filter, the matched filter operation and diversity combining, which add up all the available SNR at each of the multipath 
components and diversity antennas. Eigenvalue decomposition provides the tools to obtain decorrelated SNR random variables. Then, the rest is repeated application of the second part to each of the decorrelated SNR random variables.

The derivation of matched filter bounds in this paper follows the same general framework of above three steps. The major contribution of the paper includes that the matched filter bounds are obtained for $q$-ary QAM signalling, where $q$ is 4, 16, and 64. In addition, the matched filter bounds are derived for fractionally-spaced channels with the reception-diversity. Thus, with a specific example of fractionally-spaced multipath-power delay profiles (MPDPs), one can readily obtain the matched filter bounds and compare with their computer-simulation results.

Organization of the paper is as follows: Section II. provides system description. Section III. describes the detailed derivation of matched filter bound. Section IV. discusses three cases of interests. The three areas are 1) a single ISI channel case, 2) maximal ratio combining case, and 3) receive diversity-channels, each of the channel being ISI. Section V. provides concluding remarks.

\section{SYSTEM DESCRIPTION}

Figure -1 describes the underlying channel and matched filter system. $q$-ary QAM symbols are generated and pulse-shaped by the transmit filter $f(t)$. The transmitted signal propagates through the wireless channel and arrive to the space-diversity antennas at the receiver. At each antenna branch, the independent wireless channel is modelled as filters $\left\{b^{l}(t), l=1, \ldots, L\right\}$. Upon receiving the signal, the optimal receiver performs matched filtering at each branch and samples at the optimum sampling point. The detection performance will be evaluated on the sample of the received signal. Before starting with the derivations, let us describe some of the important assumptions we make in the derivation:

- Assumption 1: Matched filter bound is based on a single-shot symbol transmission and detection such that it ignores any intersymbol interference.

- Assumption 2: The matched filter theory holds with the colored noise; however in this paper we assume that the noise is complex-valued white Gaussian.

- Assumption 3: The channel is assumed to be time-invariant over the duration of the overall pulse, which includes the channel and the transmit shaping filter (the anti-aliasing receive filter as well).

- Assumption 4: The transmit shaping filter is assumed to employ an excess bandwidth of less 
than $100 \%$, and thus the channel can be modelled as a half-symbol spaced finite impulse response filter without loss of information.

- Assumption 5: The half-spaced fading components of the channel are mutually uncorrelated, (i.e., the wide-sense stationary uncorrelated scattering assumption).

\section{A. Single-shot system equation}

Based on the assumptions made, we first define the basic equation for the receive signal in a single channel case. The received signal for a single-shot transmission of a pulse modulated by the information symbol $I_{0}$ can be written as

$$
x_{s}(t)=\sum_{p=0}^{N_{R}-1} b_{p} f\left(t-p T_{B}\right) I_{0}+n(t)=h(t) I_{0}+n(t),
$$

where

- $I_{0}$ denotes the $q$-ary QAM symbol

- $b_{p}$ denotes the $p$-th component of the half symbol-spaced finite impulse response (FIR) filter of the channel at a fixed instant of time, $p=0,1, \ldots, N_{R}-1$ (we use $N_{R}=3$ for simulation examples in this paper).

- The half-symbol spaced multipath power delay profile (MPDP) is defined as

$$
\begin{aligned}
\phi_{c}(p) & =E\left\{b_{p}^{*} b_{q}\right\} \\
& =\alpha_{p}^{2} \delta(p-q)
\end{aligned},
$$

and thus $\alpha_{p}^{2}$ denotes the average power of the $p$-th component.

- $f(t)$ is a square root raised cosine filter, and $F(\omega)$ denotes the Fourier transform of $f(t)$,

$$
F(\omega)=\int_{-\infty}^{\infty} f(t) \exp (-j \omega t) d t
$$

- $\quad n(t)$ denotes the zero-mean, complex-valued additive white Gaussian noise with two-side power spectral density $N_{o}$.

- $h(t)$ denotes the signal part of received signal $-\infty<t<\infty$ due to the single-shot transmission of the symbol $I_{0}$ at $t=0^{\dagger}$,

Now consider the Fourier transform of $h(t)$, which is denoted as

$$
H(\omega)=F\{h(t)\}=F(\omega) \sum_{p=0}^{N_{R}-1} b_{p} \exp \left(-j \omega p \frac{T_{B}}{2}\right)
$$

\footnotetext{
$\dagger$. A non-causal representation of transmission and reception of the signal is used for brevity.
} 
where $T_{B}$ denotes the symbol-period. Then, the complex-conjugate $H^{*}(\omega)$ can be written as

$$
H^{*}(\omega)=F^{*}(\omega) \sum_{p=0}^{N_{R}-1} b_{p}^{*} \exp \left(j \omega p \frac{T_{B}}{2}\right) .
$$

Based on the matched filter theory [1], $H^{*}(\omega)$ is the optimal filter that maximizes the detection SNR. Now applying the matched filter response $H^{*}(\omega)$ to the received signal $x(t)$, we have the matched filtered signal which can be written in the Fourier transform domain as

$$
H^{*}(\omega) X(\omega)=H^{*}(\omega) H(\omega) I_{0}+H^{*}(\omega) N_{o} .
$$

The inverse Fouier transform of (6) provides the time-domain response of the matched filtered signal. Now notice that the autocorrelation function became the overall channel. It is the inverse Fouier transform of $\left\{H^{*}(\omega) H(\omega)\right\}, \frac{1}{2 \pi} \int_{-\infty}^{\infty}\left\{H^{*}(\omega) H(\omega)\right\} \exp (j \omega \tau) d \omega$, and is Hermitian symmetric around $\tau=0$. Thus, by sampling the matched filtered output response at $\tau=0$, we achieve the optimal matched filter output which we discussed in the next sub-section.

\section{B. Sampled, matched filter output}

Now let $z_{s}$ denote the receive signal sample at $\tau=0$, and then for the detection of $I_{0}$ the following equation provides the sufficient statistic

$$
z_{s}=A_{s} I_{0}+v_{s}
$$

where

- $A_{s}$ denotes the zeroth lag value of the Hermitian symmetric autocorrelation channel, which is the value of autocorrelation function at time $\tau=0$. It is a random variable and implies the instantaneous energy of the cascade response, the channel and transmit-shaping filters, and can be written as

$$
\begin{aligned}
A_{s} & =\left.\frac{1}{2 \pi} \int_{-\infty}^{\infty} H^{*}(\omega) H(\omega) \exp (j \omega \tau) d w\right|_{\tau=0}=\frac{1}{2 \pi} \int_{-\infty}^{\infty} H^{*}(\omega) H(\omega) d \omega \\
& =\frac{1}{2 \pi} \int_{-\infty}^{\infty} F^{*}(\omega) F(\omega) \sum_{p=0}^{N_{R}-1} b_{p}^{*} \exp \left(j \omega p \frac{T_{B}}{2}\right) \sum_{q=0}^{N_{R}-1} b_{q} \exp \left(-j \omega q \frac{T_{B}}{2}\right) d \omega
\end{aligned}
$$

From the uncorrelated scattering assumption (Assumption 5), (8) can be written as

$$
\begin{aligned}
A_{s} & =\sum_{p=0}^{N_{R}-1} \sum_{q=0}^{N_{R}-1} b_{p}^{*} b_{q} \frac{1}{2 \pi} \int_{-\infty}^{\infty}|F(\omega)|^{2} \exp \left(-j \omega(q-p) \frac{T_{B}}{2}\right) d \omega \\
& =\sum_{p=0}^{N_{R}-1} \sum_{q=0}^{N_{R}-1} b_{p}^{*} b_{q} f_{r c}\left(t=(q-p) \frac{T_{B}}{2}\right)
\end{aligned}
$$


where $f_{r c}(t)$ is the raised cosine filter response,

- $I_{0}$ denotes the transmitted $q$-ary QAM symbol, $E\left(I_{0}\right)=0.0$ and

$$
\operatorname{Var}\left(I_{0}\right)=\frac{2(q-1)}{3}
$$

- $v_{s}$ denotes the matched filtered noise output sampled at $t=0$ which is

$$
v_{s}=\left.\int_{-\infty}^{\infty} n(\tau) h(t-\tau) d \tau\right|_{t=0}
$$

thus $v_{s}$ is zero-mean with $\operatorname{Var}\left(v_{s}\right)=N_{0} \cdot A_{s}$.

It is worthwhile to note that (7) provides sufficient information required to compute the detection probability of the single-shot matched filter receiver. Also note that $A_{s}$ denotes the random variable representing the instantaneous energy of the cascade of filters which are the transmit shaping filter, the channels at all diversity branches at a specific time instant.

\section{MATCHED FILTER BOUND CALCULATION}

\section{A. Square-QAM symbol error probability}

In this section, for a particular values of $A_{s}$ and $N_{o}$ the symbol error probability will be

evaluated for square $q$-ary QAM signaling, i.e. $q=2^{k}$ where $k$ is even. Referring to Figure -2 , we start with summary of the following relationships which become useful in later sections:

- The average energy of the square-QAM signaling set can be computed as, using (10) and the definition given in Figure -2

$$
E_{s}=E\left(I_{0}^{2}\right)\left(\frac{A_{s}}{\sqrt{2}}\right)^{2}=\frac{2(q-1)}{3}\left(\frac{A_{s}}{\sqrt{2}}\right)^{2}=\frac{q-1}{3} A_{s}^{2} .
$$

- The minimum Euclidean distance of the square-QAM constellation is

$$
d_{\min }=\sqrt{2} \cdot A_{s} .
$$

- The instantaneous signal to noise ratio is

$$
\frac{\text { signal power }}{\text { noise power }}=\gamma=k \cdot \gamma_{b}=\frac{E_{s}}{A_{s} \cdot N_{o}}=\frac{(q-1)}{3} \frac{A_{s}}{N_{o}} \text {, }
$$

where $\gamma$ is the instantaneous SNR, $k=\log _{2}(\sqrt{q})$ the number of bits per symbol, $\gamma_{b}$ is the instantaneous SNR/bit.

Then, the $q$-ary square-QAM symbol error probability at a particular channel gain $A_{s}=a$, can 
be computed as

$$
P_{q}\left(A_{s}=a\right)=2\left(1-\frac{1}{\sqrt{q}}\right) \operatorname{erfc}\left(\frac{d_{\text {min }}(a)}{2 \sqrt{\operatorname{Var}\left(v_{s}\right)}}\right) \cdot\left\{1-\frac{1}{2}\left(1-\frac{1}{\sqrt{q}}\right) \operatorname{erfc}\left(\frac{d_{\text {min }}(a)}{2 \sqrt{\operatorname{Var}\left(v_{s}\right)}}\right)\right\} .
$$

(15) can be tightly upper-bounded by the first term, (16). Figure -3 provides the comparison of (15) and (16). We note that the approximation is asymptotically efficient and very tight even at low SNR region. Thus, we will use the following approximation

$$
P_{q}(a) \approx 2\left(1-\frac{1}{\sqrt{q}}\right) \operatorname{erfc}\left(\frac{d_{\min }(a)}{2 \sqrt{a N_{o}}}\right) \text {. }
$$

Now, solving for $a$ in (12), i.e., $a^{2}=\frac{3}{(q-1)} E_{s}$ we have

$$
d_{\text {min }}(a)=\sqrt{2} a=\sqrt{\frac{2 \cdot 3}{(q-1)} E_{s}},
$$

but using (14), (17) is

$$
d_{\text {min }}(a)=\sqrt{\frac{2 \cdot 3}{(q-1)} a k \gamma_{b} N_{o}},
$$

Then, the approximation of the symbol error probability (16) can be written as

$$
P_{q}(a)=2\left(1-\frac{1}{\sqrt{q}}\right) \operatorname{erfc}\left(\sqrt{\frac{3}{2(q-1)} k \gamma_{b}}\right)
$$

or simply

$$
P_{q}(a)=2\left(1-\frac{1}{\sqrt{q}}\right) \operatorname{erfc}\left(\sqrt{\frac{a}{2 N_{o}}}\right) .
$$

We will use this approximation to derive the averaged symbol error probability.

\section{B. Average symbol error probability for square-QAM}

Now, the symbol error probability, averaged over the ensemble of the channel or equivalently that of $A_{s}$, can be computed from

$$
\overline{P_{q}}\left(\overline{\gamma_{b}}\right)=\int_{0}^{\infty} P_{q}(a) \operatorname{Pr}\left(A_{s}=a\right) d a,
$$

where $\overline{P_{q}}\left(\overline{\gamma_{b}}\right)$ denotes the averaged symbol error probability of $q$-ary QAM system for the average 
input SNR which is defined as

$$
\overline{\gamma_{b}}=E\left\{\gamma_{b}\right\}=\frac{(q-1)}{3 k N_{o}} E\left\{A_{s}\right\}
$$

Thus, we need to know the distribution function of the random variable $A_{s}$.

From (9), we may note that the random variable can be written as follows, using $N_{R}=3$ for simplicity of illustration:

$$
A_{s}=\left(\begin{array}{lll}
b_{0}^{*} & b_{1}^{*} & b_{2}^{*}
\end{array}\right)\left[\begin{array}{ccc}
f_{r c}(0) & f_{r c}\left(T_{B} / 2\right) & f\left(T_{B}\right) \\
f_{r c}\left(T_{B} / 2\right) & f_{r c}(0) & f_{r c}\left(T_{B} / 2\right) \\
f\left(T_{B}\right) & f_{r c}\left(T_{B} / 2\right) & f_{r c}(0)
\end{array}\right]\left(\begin{array}{l}
b_{0} \\
b_{1} \\
b_{2}
\end{array}\right) \text {. }
$$

Denote the matrix in the middle as $\mathbf{F}_{r c}$, where $f_{r c}(t)$ denote the raised cosine function. Now, we represent each of the fading channel tap as $b_{i}=\alpha_{i} \rho_{i}$, multiplication of an attenuation factor and the unit-variance, complex-valued Gaussian random variable $\rho_{i}$. Therefore, we can write the channel vector $\mathbf{b}$ as

$$
\mathbf{b}=\alpha \rho=\left(\begin{array}{cc}
\alpha_{0} & 0 \\
\alpha_{1} & \\
0 & \alpha_{2}
\end{array}\right)\left(\begin{array}{l}
\rho_{0} \\
\rho_{1} \\
\rho_{2}
\end{array}\right)
$$

where $E\left(\rho \rho^{H}\right)=\Xi_{N_{R} \times N_{R}}$, a $3 \times 3$ identity matrix because $\rho_{i}, i=0,1,2$ are assumed to be mutually uncorrelated.

Using (24), (23) can now be rewritten,

$$
\begin{aligned}
A_{s} & =\mathbf{b}^{H} \mathbf{F}_{r c} \mathbf{b}=\rho^{H} \alpha^{H} \mathbf{F}_{r c} \alpha \rho, \\
& =\rho^{H} \mathbf{G} \rho
\end{aligned}
$$

where in the second line we have defined $\mathbf{G}=\alpha^{H} \mathbf{F}_{r c} \alpha$. It is important to note that for a fixed MPDP, $\mathbf{G}$ is fixed. Also note that $\mathbf{G}$ is Hermitian symmetric. In addition, since $A_{s}$ is the energy of the cascade filter (8) it is non-negative definite. For any non-negative definite Hermitian symmetric matrix $\mathbf{G}$, there exist an orthonormal matrix $\mathbf{Q}$ such that $\mathbf{Q G} \mathbf{Q}^{H}=\Lambda$, or

$$
\mathbf{G}=\mathbf{Q}^{H} \Lambda \mathbf{Q}
$$

where $\Lambda$ is a diagonal matrix with the diagonal elements, $\lambda_{p} \geq 0, p=0,1,2$, being the eigenvalues of the matrix $\mathbf{G}$. 
Now rewriting (25) using (26) we have

$$
A_{s}=\rho^{H} \mathbf{G} \rho=\rho^{H} \mathbf{Q}^{H} \Lambda \mathbf{Q} \rho=\dot{\rho}^{H} \Lambda \dot{\rho}=\sum_{p=0}^{N_{R}-1} \lambda_{p}\left|\dot{\rho}_{p}\right|^{2},
$$

where we have now defined $\dot{\rho}=\mathbf{Q} \rho$. Note that $\dot{\rho}_{p}, p=0,1,2$ are again mutually independent,

complex-valued Gaussian random number with zero-mean and unit-variance, and thus $\lambda_{p}\left|\rho_{p}\right|^{2}$, $p=0,1,2$, are iid $\chi^{2}$-distributed random variables with the characteristic function $1 /\left(1-j v \lambda_{p}\right)$. Thus, the characteristic function of $A_{s}$ is the product terms of

$$
E\left\{\exp \left(j v A_{s}\right)\right\}=\prod_{p=0}^{2} \frac{1}{\left(1-j v \lambda_{p}\right)} .
$$

\section{THREE CASES OF INTERESTS}

We now want to evaluate the average probability of symbol errors. For easy of illustration, we divide the tasks based on three cases of interests. The first case is for a single, ISI channel case, where $A_{s}$ is expected to be represented with $N_{R}$ distinctive eigenvalues. The second case is for the case of $L$-diversity antennas, with flat-fading channel, where $A_{s}$ can be represented with a single, $L$-repeated eigenvalue. The third case is combination of the first two, where $A_{s}$ is represented with $L$-repeated set of $N_{R}$-distinct eigenvalues. With the supply of input values such as MPDPs, the shaping filter characteristics and the averaged input SNR, the derivation in this section allows us to obtain the averaged, square-QAM symbol error probabilities as function of averaged input SNR.

\section{A. Distinct eigenvalues (no eigenvalues in multiplicity)}

When all the eigenvalues are distinct, (28) can be expressed as

$$
E\left\{\exp \left(j v A_{s}\right)\right\}=\sum_{p=0}^{N_{R}-1} \frac{\pi_{p}}{1-j v \lambda_{p}}
$$

where we have defined the weight of an individual random variable to be

$$
\pi_{p}=\prod_{\substack{q=0 \\ q \neq p}}^{N_{R}-1} \frac{1}{\left(1-\lambda_{q} / \lambda_{p}\right)}
$$

Finally, we can write the probability density function for $A_{s}$ which is the weighted sum of $N_{R} \chi^{2}$ distributed random variables. That is,

$$
\operatorname{Pr}\left(A_{s}=a\right)=\sum_{p=0}^{N_{R}-1} \pi_{p} \frac{e^{-a / \lambda_{p}}}{\lambda_{p}} .
$$


Now substituting (31) and (20) into (21) we have

$$
\begin{aligned}
\overline{P_{q}}\left(\overline{\gamma_{b}}\right) & =\int_{0}^{\infty} P_{q}(a) \operatorname{Pr}\left(A_{s}=a\right) d a \\
& =2\left(1-\frac{1}{\sqrt{q}}\right) \sum_{p=0}^{N_{R}-1} \pi_{p} \int_{0}^{\infty} \operatorname{erfc}\left(\sqrt{\frac{a}{2 N_{o}}}\right) \frac{e^{-a / \lambda_{p}}}{\lambda_{p}} d a
\end{aligned}
$$

Now define

$$
Y=\frac{A_{s}}{2 N_{o}}
$$

then by change of variable (32) can be rewritten

$$
\overline{P_{q}}\left(\overline{\gamma_{b}}\right)=4\left(1-\frac{1}{\sqrt{q}}\right)\left\{\frac{1}{2} \sum_{p=0}^{N_{R}-1} \pi_{p} \int_{0}^{\infty} \operatorname{erfc}(\sqrt{y}) \frac{e^{-y / \lambda_{p}}}{\dot{\lambda}_{p}^{\cdot}} d y\right\},
$$

where we have defined for $p=0,1, \ldots, N_{R}-1, \dot{\lambda}_{p}=\frac{\lambda_{p}}{2 N_{o}}$. Note that the weight terms $\pi_{p}$ stays the same. Then, (34) becomes

$$
\overline{P_{q}}\left(\overline{\gamma_{b}}\right)=4\left(1-\frac{1}{\sqrt{q}}\right)\left(\frac{1}{2} \sum_{i=0}^{N_{R}-1} \pi_{i}\left(1-\sqrt{\frac{\dot{\lambda}_{i}}{1+\dot{\lambda}_{i}}}\right)\right),
$$

where the relationship between the average $\mathrm{SNR} / \mathrm{bits}$ and the eigenvalues are

$$
\overline{\gamma_{b}}=\frac{2(q-1)}{3 k} \frac{E\left\{A_{s}\right\}}{2 N_{o}}=\frac{2(q-1)}{3 k} E\{Y\}=\frac{2(q-1)}{3 k}\left(\frac{1}{2 N_{o}}\right) \sum_{i=o}^{N_{R}-1} \lambda_{i} .
$$

Now, the following steps describe the procedure of how to compute the matched filter symbol error probability bounds when the input parameters are the average SNR/bits $\overline{\gamma_{b}}$, the constellation size $q$ and the multipath power delay profile.

- Evaluate the eigenvalues $\left\{\lambda_{i}, i=0,1, \ldots, N_{R}\right\}$ using given MPDP and the transmit shaping filter, which is described in (23) to (27).

- Now determine the value of $\left(\frac{1}{2 N_{o}}\right)$ for the given value of $\bar{\gamma}_{b}$ and $q$ by

$$
\frac{1}{2 N_{o}}=\frac{3 \bar{\gamma}_{b} \log _{2} q}{2(q-1) \sum_{i=o}^{N_{R}-1} \lambda_{i}}
$$

- Calculate $\left\{\dot{\lambda}_{i}, i=0,1, \ldots, N_{R}\right\}$ by evaluating

$$
\dot{\lambda}_{p}=\frac{\lambda_{p}}{2 N_{o}}
$$


- Finally, substitute (38) into (35) to calculate the average symbol error probability.

\section{B. Eigenvalues occurring in multiplicity}

We now consider the case of $L$-times repeated eigenvalues, i.e.

$$
E\left\{\exp \left(j v A_{s}\right)\right\}=\left(\frac{1}{1-j v \lambda_{1}}\right)^{L} .
$$

This is the case when we have equal gain, independent diversity sources. Then, (27) takes the expression

$$
A_{s}=\sum_{p=0}^{L-1} \lambda_{1}\left|\dot{\rho}_{p}\right|^{2}
$$

where $\left|\dot{\rho}_{p}\right|^{2}$ again are iid Chi-square distribution with unit mean. The distribution function for this case is $\operatorname{Pr}\left(A_{s}=a\right)=\frac{1}{(L-1) ! \lambda_{1}^{L}} a^{L-1} e^{-a / \lambda_{1}}$. Then, the average symbol error probability is

$$
\begin{aligned}
\overline{P_{q}}\left(\overline{\gamma_{b}}\right) & =\int_{0}^{\infty} P_{q}(a) \operatorname{Pr}\left(A_{s}=a\right) d a \\
& =4\left(1-\frac{1}{\sqrt{q}}\right) \int_{0}^{\infty} \frac{1}{2} \operatorname{erfc}\left(\sqrt{\frac{a}{2 N_{o}}}\right) \frac{1}{(L-1) ! \lambda_{1}^{D}} a^{L-1} e^{-a / \lambda_{1}} d a .
\end{aligned}
$$

Then, by defining $Y=A_{s} /\left(2 N_{o}\right)$, we have

$$
\overline{P_{q}}\left(\overline{\gamma_{b}}\right)=4\left(1-\frac{1}{\sqrt{q}}\right) \int_{0}^{\infty} \frac{1}{2} \operatorname{erfc}(\sqrt{y}) \frac{1}{(L-1) ! \dot{\lambda}_{1}^{L}} y^{L-1} e^{-y / \dot{\lambda}_{1}} d y
$$

where we again defined $\dot{\lambda}_{1}=\lambda_{1} /\left(2 N_{o}\right)$. Then, we obtain ${ }^{\dagger}$

$$
\overline{P_{q}}\left(\overline{\gamma_{b}}\right)=4\left(1-\frac{1}{\sqrt{q}}\right)\left(\frac{1-\Omega}{2}\right)^{L} \sum_{k=0}^{L-1}\left(\begin{array}{c}
L-1+k \\
k
\end{array}\right)\left(\frac{1+\Omega}{2}\right)^{k}
$$

where we defined $\Omega=\sqrt{\dot{\lambda}_{1} /\left(1+\dot{\lambda}_{1}\right)}$.

Now, following steps describe the procedure to compute the averaged symbol error probability with the input parameters of the average SNR/bits $\overline{\gamma_{b}}$, the constellation size $q$ and $L$ diversity paths of equal gain $\lambda_{1}$.

- Now determine the value of $\frac{1}{2 N_{o}}$ for the given value of $\overline{\gamma_{b}}$ and $q$ by

$$
\frac{1}{2 N_{o}}=\frac{3 \overline{\gamma_{b}} \log _{2} q}{2(q-1) D \lambda_{1}}
$$

- Compute $\dot{\lambda}_{1}=\lambda_{1} /\left(2 N_{o}\right)$ and $\Omega=\sqrt{\dot{\lambda_{1}} /\left(1+\dot{\lambda}_{1}\right)}$

$$
\dagger \text {. using } \int_{0}^{\infty} \frac{1}{2} \operatorname{erfc}(\sqrt{y}) \frac{1}{(L-1) ! \dot{\lambda}_{1}^{L}} y^{L-1} e^{-y / \dot{\lambda}_{1}} d y=\left(\frac{1-\Omega}{2}\right)^{L} \sum_{k=0}^{L-1}\left(\begin{array}{c}
L-1+k \\
k
\end{array}\right)\left(\frac{1+\Omega}{2}\right)^{k}[6] \text {. }
$$


- Finally, substitute $\Omega$ into (43) to calculate the average symbol error probability

The considered situation is when each diversity branch is modelled as a single Rayleigh fading tap channels. Then, the matched filter combiner simply becomes the maximal ratio combining of the received signal. Figure -4 Figure -5 Figure -6 are the matched filter bounds for $q$-QAM with $L$-diversity antenna reception of the signal, for $q$ equal to 4, 16 and 64 . As the order of diversity increases, we note that the matched filter bounds of $L$-diversity channels approach the SER performance of the AWGN channel.

\section{Combination of distinct and multiple poles}

We now consider the case where there is $L$ diversity antenna reception of the signal and each channel is a multipath ISI channel. Now, the instantaneous channel gain $A_{s}$ can be written as

$$
A_{s}=\sum_{l=0}^{L-1} \sum_{p=0}^{N_{R}-1} \lambda_{p}\left|\rho_{l, p}\right|^{2},
$$

where $\dot{\rho_{l, p}}, p=0,1, \ldots, N_{R}$ are mutually independent, complex-valued Gaussian random number with zero-mean and unit-variance, and thus $\lambda_{p}\left|\rho_{l, p}\right|^{2}$ are iid $\chi^{2}$-distributed random variables. Note that the MPDP stays the same for each of different antennas, and thus the same set of $N_{R}$ (distinct) eigenvalues should be repeating $L$ times. Thus, the characteristic function becomes

$$
E\left\{\exp \left(j v A_{s}\right)\right\}=\prod_{p=0}^{N_{R}-1} \frac{1}{\left(1-j v \lambda_{p}\right)^{L}} .
$$

Now, for the example of $L=2$ and $N_{R}=3$, by the method of partial fraction expansion (46) can be decomposed into

$$
\prod_{p=0}^{2} \frac{1}{\left(1-j \vee \lambda_{p}\right)^{2}}=\sum_{p=0}^{2}\left(\frac{\Gamma_{2, p}}{\left(1-j \vee \lambda_{p}\right)^{2}}+\frac{\Gamma_{1, p}}{1-j \vee \lambda_{p}}\right),
$$

where $\Gamma$ values are the expansion coefficients. Then, the probability density function is

$$
\operatorname{Pr}\left(A_{s}=a\right)=\sum_{p=0}^{2}\left(\Gamma_{1, p} \frac{e^{-a / \lambda_{p}}}{\lambda_{p}}+\Gamma_{2, p} \frac{1}{(L-1) ! \lambda_{p}^{L}} a^{L-1} e^{-a / \lambda_{p}}\right) .
$$


Then, the average symbol error probability is

$$
\begin{aligned}
& \overline{P_{q}}\left(\overline{\gamma_{b}}\right)=\int_{0}^{\infty} P_{q}(a) \operatorname{Pr}\left(A_{s}=a\right) d a \\
& =\int_{0}^{\infty} 2\left(1-\frac{1}{\sqrt{q}}\right) \operatorname{erfc}\left(\sqrt{\frac{a}{2 N_{o}}}\right)\left(\sum_{p=0}^{2}\left(\Gamma_{1, p} \frac{e^{-a / \lambda_{p}}}{\lambda_{p}}+\Gamma_{2, p} \frac{1}{(L-1) ! \lambda_{p}^{L}} a^{L-1} e^{-a / \lambda_{p}}\right)\right) d a \\
& =4\left(1-\frac{1}{\sqrt{q}}\right) \sum_{p=0}^{2}\left[\Gamma_{1, p} P_{1}\left(\dot{\lambda}_{p}\right)+\Gamma_{2, p} P_{2}\left(\dot{\lambda}_{p}\right)\right]
\end{aligned}
$$

where we have defined

- $\left.P_{1}\left(\dot{\lambda}_{p}\right)=\frac{1}{2}\left(1-\sqrt{\dot{\lambda}_{p} /\left(1+\dot{\lambda}_{p}\right.}\right)\right)$

- $P_{2}\left(\dot{\lambda}_{p}\right)=((1-\Omega) / 2)^{L} \sum_{i=0}^{L-1}\left(\begin{array}{c}L-1+i \\ i\end{array}\right)((1+\Omega) / 2)^{i}$,

- $\dot{\lambda}_{p}=\lambda_{p} /\left(2 N_{o}\right)$

Now, the following steps describe how to compute the average probability given the MPDP, the number of diversity channel $L$, the average SNR/bits $\overline{\gamma_{b}}$ and the constellation size $q$ :

- Define the average SNR/bits (note, this is not the average SNR/bits/channel),

$$
\overline{\gamma_{b}}=\frac{2(q-1)}{3 k} \frac{E\left\{A_{s}\right\}}{2 N_{o}}=\frac{2(q-1)}{3 k} E\{Y\}=\frac{2(q-1)}{3 k}\left(\frac{1}{2 N_{o}}\right) L \sum_{i=o}^{N_{R}-1} \lambda_{i}
$$

- Evaluate the eigenvalues $\left\{\lambda_{i}, i=0,1, \ldots, N_{R}\right\}$ for given $L$, MPDP and the transmit shaping filter, taking the same approach as (23) to (27).

- Determine the value of $1 /\left(2 N_{o}\right)$ for the given value of $\overline{\gamma_{b}}, L$ and $q$ by

$$
\frac{1}{2 N_{o}}=\frac{3 \bar{\gamma}_{b} \log _{2} q}{2(q-1) \cdot L \cdot \sum_{i=o}^{N_{R}-1} \lambda_{i}}
$$

- Calculate $\left\{\dot{\lambda}_{i}, i=0,1, \ldots, N_{R}\right\}$ by evaluating

$$
\dot{\lambda}_{p}=\frac{\lambda_{p}}{2 N_{o}}
$$

- Finally, substitute (52) into (49) to calculate the average symbol error probability

Figure -7, Figure -8 and Figure -9 show the matched filter bounds for $q$-QAM transmission, $q=4,16$, and 64 respectively, over the multipath fading frequency-selective channels. The multipath power delay profiles we used are MPDP-1 $=\left(\begin{array}{lll}0.7413 & 0.23430 .0234\end{array}\right)$ and MPDP-2 = (0.6652 0.24470 .0900$)$. The number of diversity channels is 2 , i.e. $L=1,2$. The rms delay spreads of the two are $0.2494 T_{B}$ and $0.3257 T_{B}$ for MPDP-1 and MPDP-2 respectively. We note 
from the SEP curves that the detection performance of MPDP-2 is about $1 \sim 2 \mathrm{~dB}$ better than that of MPDP-1. This confirms the well known diversity property of the wireless channel that the larger the delay spread is the better the expected detection performance, due to inherent diversity of the delay spread channel.

\section{CONCLUDING REMARKS}

In this paper, we have derived analytical expressions for symbol error probability using the matched filter SNR for the square-QAM signals transmitted over the diversity frequency-selective channels. These theoretical bounds may not be attainable in reality due to the impractical assumptions made in deriving the bounds. Nonetheless, they provide invaluable information in designing the complex communication systems and serves as easy-to-compute analytical tools that can readily be compared with the simulation results of practical transceiver schemes. Specifically, we shall be able to observe the exact relationship between the asymptotic slopes of SER curves and influences of different shapes of MPDPs. Future work include the extension of the matched filter bounds for trellis-coded modulation cases, which will be useful to be compared with the simulation results [9].

\section{References}

[1] A.D. Whalen, Detection of Signals in Noise. San Diego: Academic Press, Inc., 1971.

[2] J.E. Mazo, "Exact matched filter bound for two-beam Rayleigh fading," IEEE Trans. Veh. Technol., vol. 39, no.7, July 1991.

[3] M.V. Clark, et. al., "Matched filter performance bounds for diversity combining receivers in digital mobile radio," IEEE Trans. Veh. Technol., vol. 41, no.4, pp. 356 - 62, Nov. 1992.

[4] F. Ling, "Matched filter-bound for time-discrete multipath Rayleigh fading channels," IEEE Trans. Veh. Technol., vol. 43, no. 2/3/4, pp. 710-713, Feb./Mar./Apr. 1995.

[5] M. Stojanovic, J. G. Proakis, and J. Catipovic, "Analysis of the impact of channel estimation errors on the performance of a decision-feedback equalizer in fading multipath channels," IEEE Trans. on Comm., vol. 43, no. 2/3/4, 877-886, Feb./Mar./Apr. 1995.

[6] J.G. Proakis, Digital Communications. New York, NY: McGraw-Hill, 3rd Edition, 1989.

[7] W.C. Jakes, ed., Microwave Mobile Communications. New York, NY: Wiley, 1974.

[8] Heung-No Lee and Gregory J. Pottie, "Fast adaptive equalization/diversity combining for 
time-varying dispersive channels," IEEE Trans. on Commun. vol. 46, pp. 1146-62, Sept. 1998.

[9] Heung-No Lee and Gregory J. Pottie, "Adaptive sequence detection using T-algorithm for multipath fading ISI channels," Proc. of IEEE International Conference on Communication (Communication Theory Mini Conference), pp. 125-9, June 1999.

[10] Heung-No Lee and Gregory J. Pottie, "Adaptive sequence detection of channel-interleaved trellis-coded modulation signals over multipath fading ISI channels," Proc. of IEEE 49 th Vehic. Tech. Conf., pp. 1474 - 9, May 1999. 


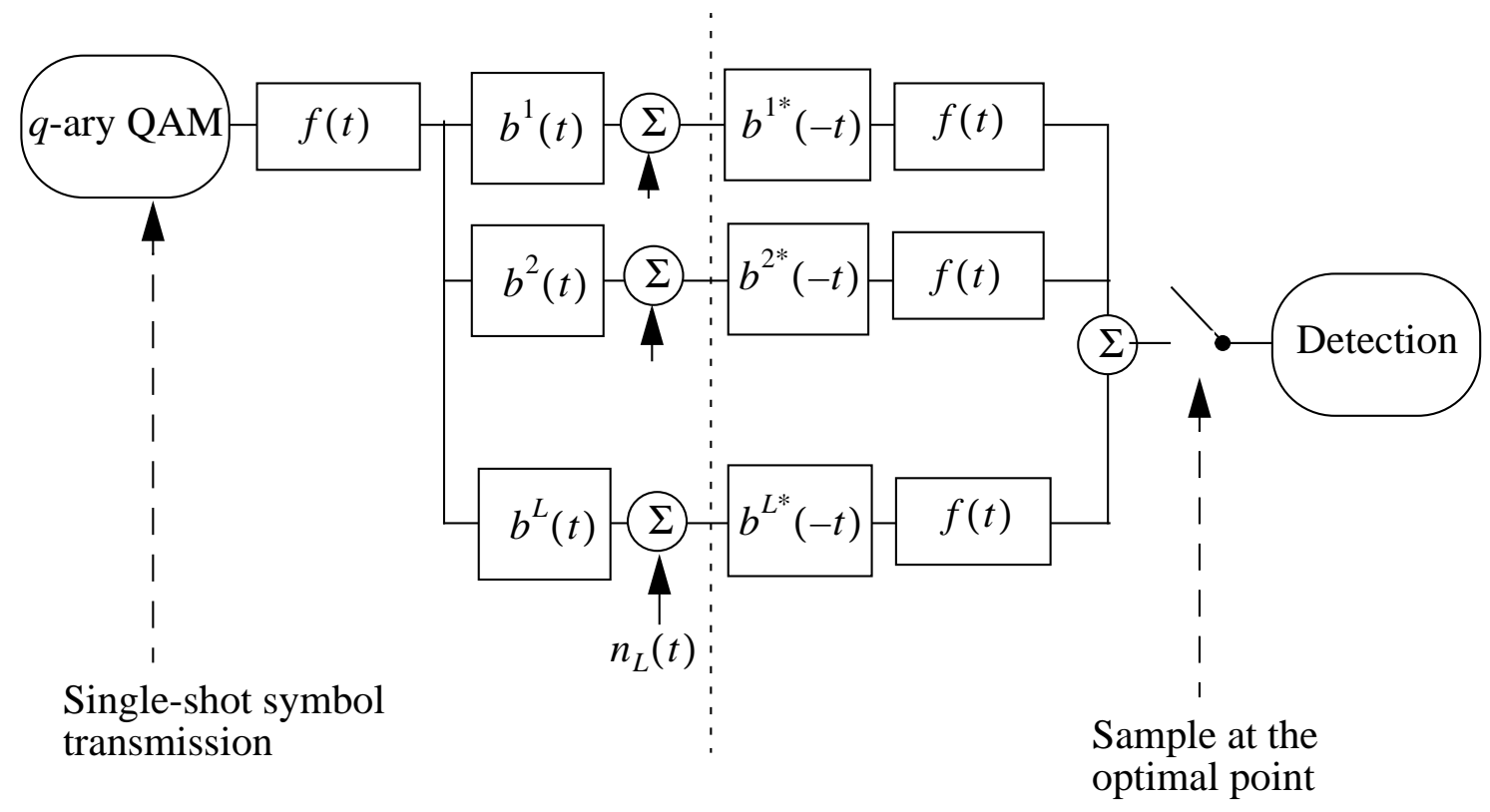

Figure -1 The diversity reception channel model, the optimal receiver structure with multiple reception of a single shot $q$-ary symbol, matched filtering, combining, singleshot sampling at the maximum SNR point, and decision

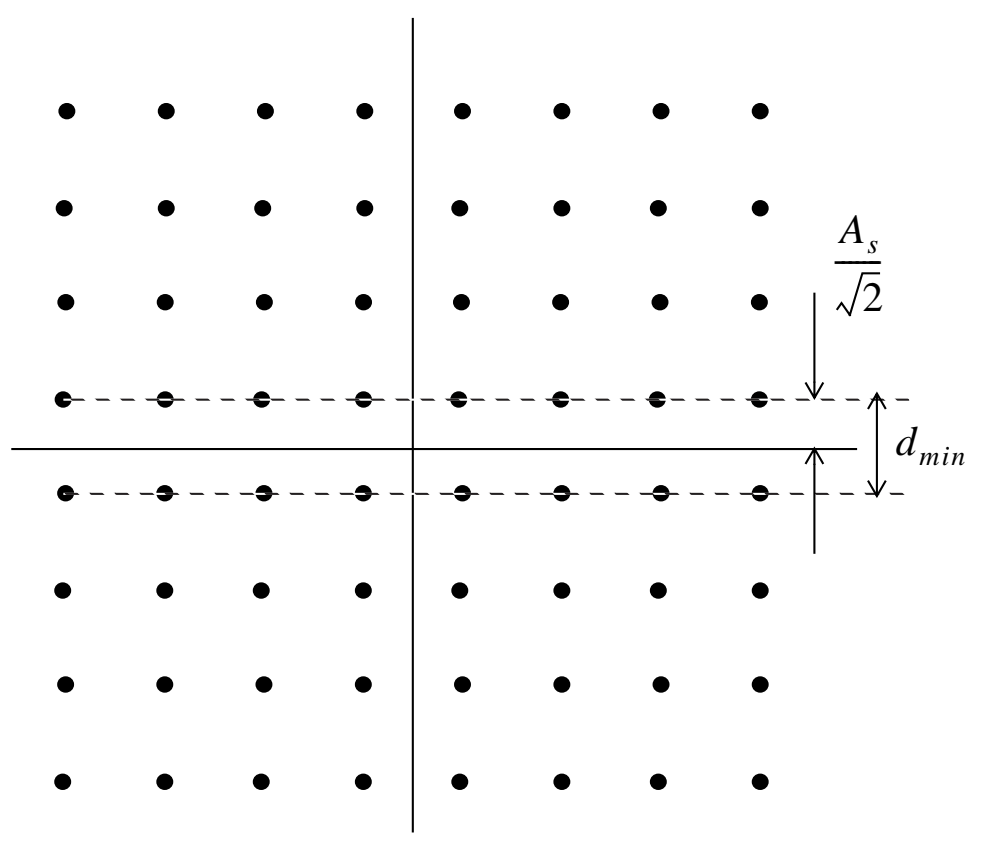

Figure -2 Illustration of received constellation with 64-QAM. $A_{s}$ denotes the instantaneous channel gain, combining all the channel paths and branches. 


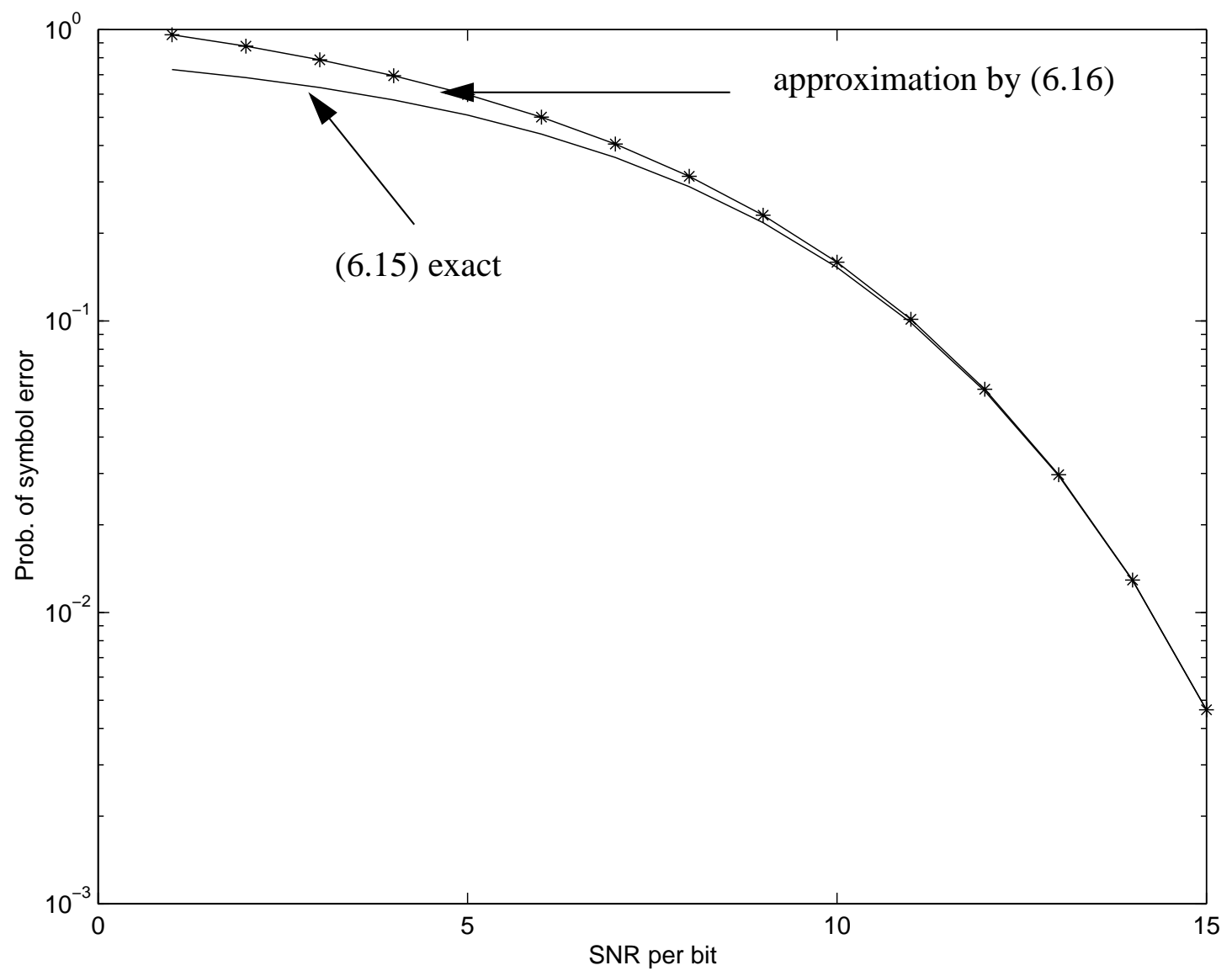

Figure -3 Illustration of upper bound of the square QAM symbol error rate 


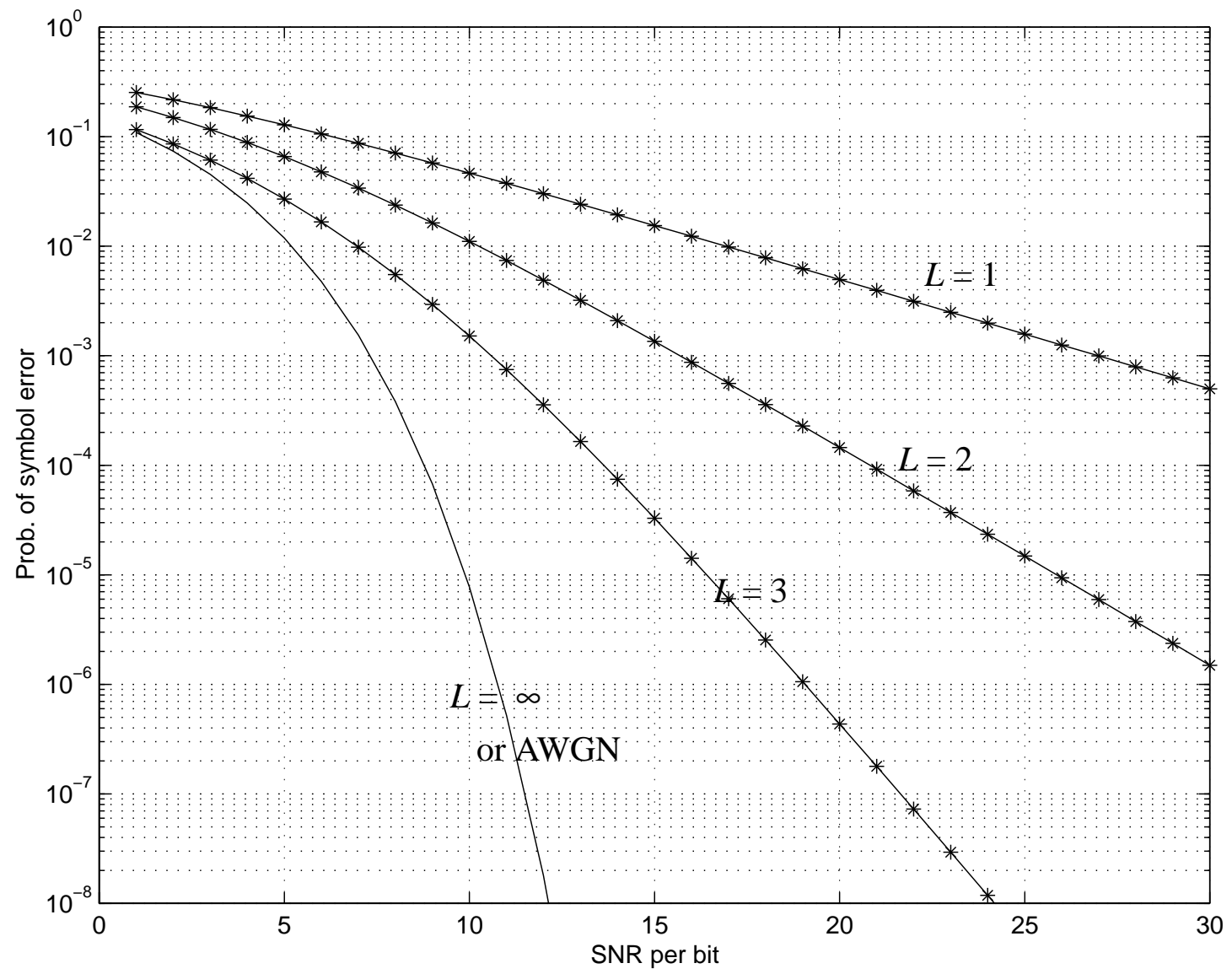

Figure -4 Matched filter bound SEP for 4-QAM transmission over $L$-flat fading diversity channels. 


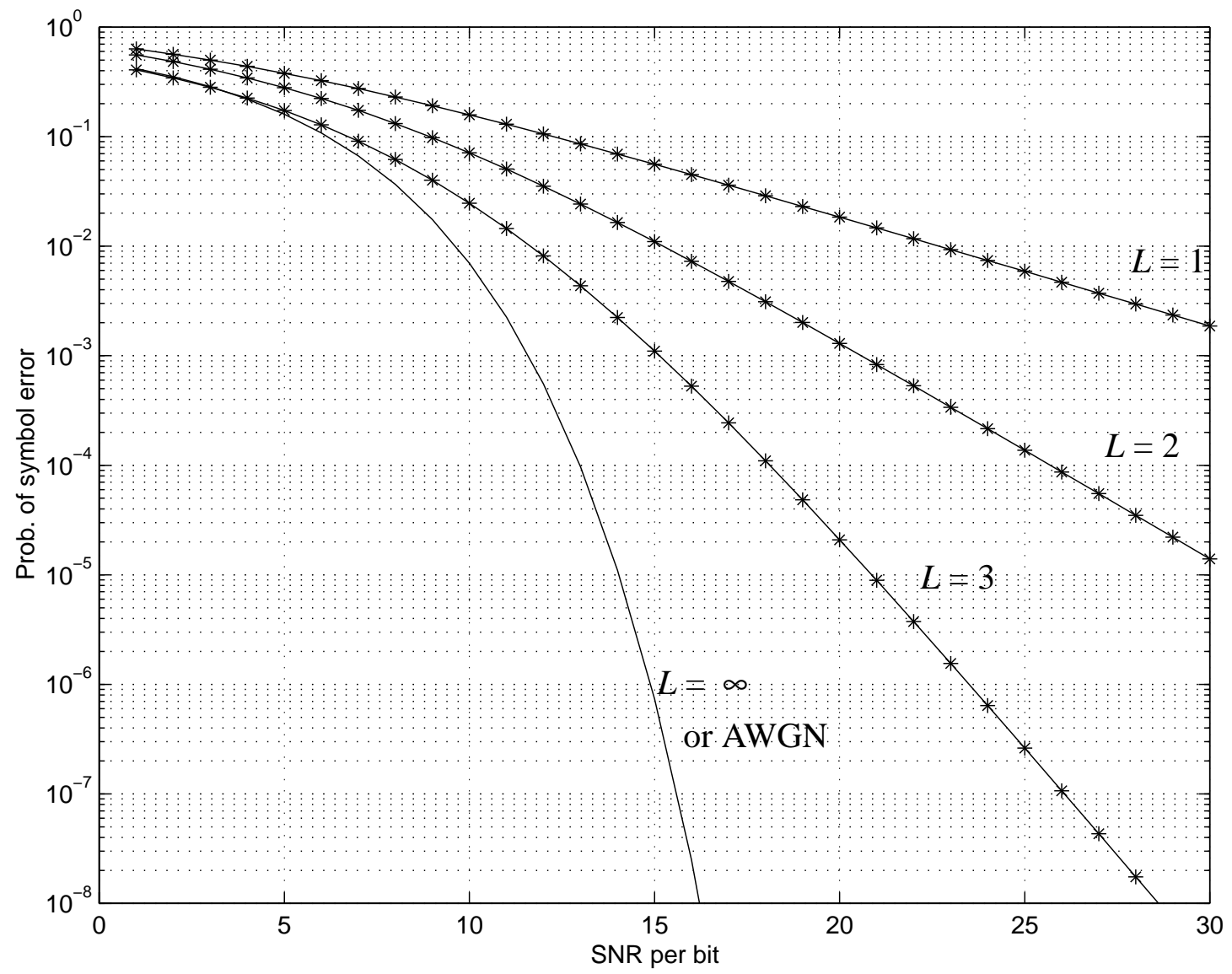

Figure -5 Matched filter bound SEP for 16-QAM transmission over $L$-flat fading diversity channels. 


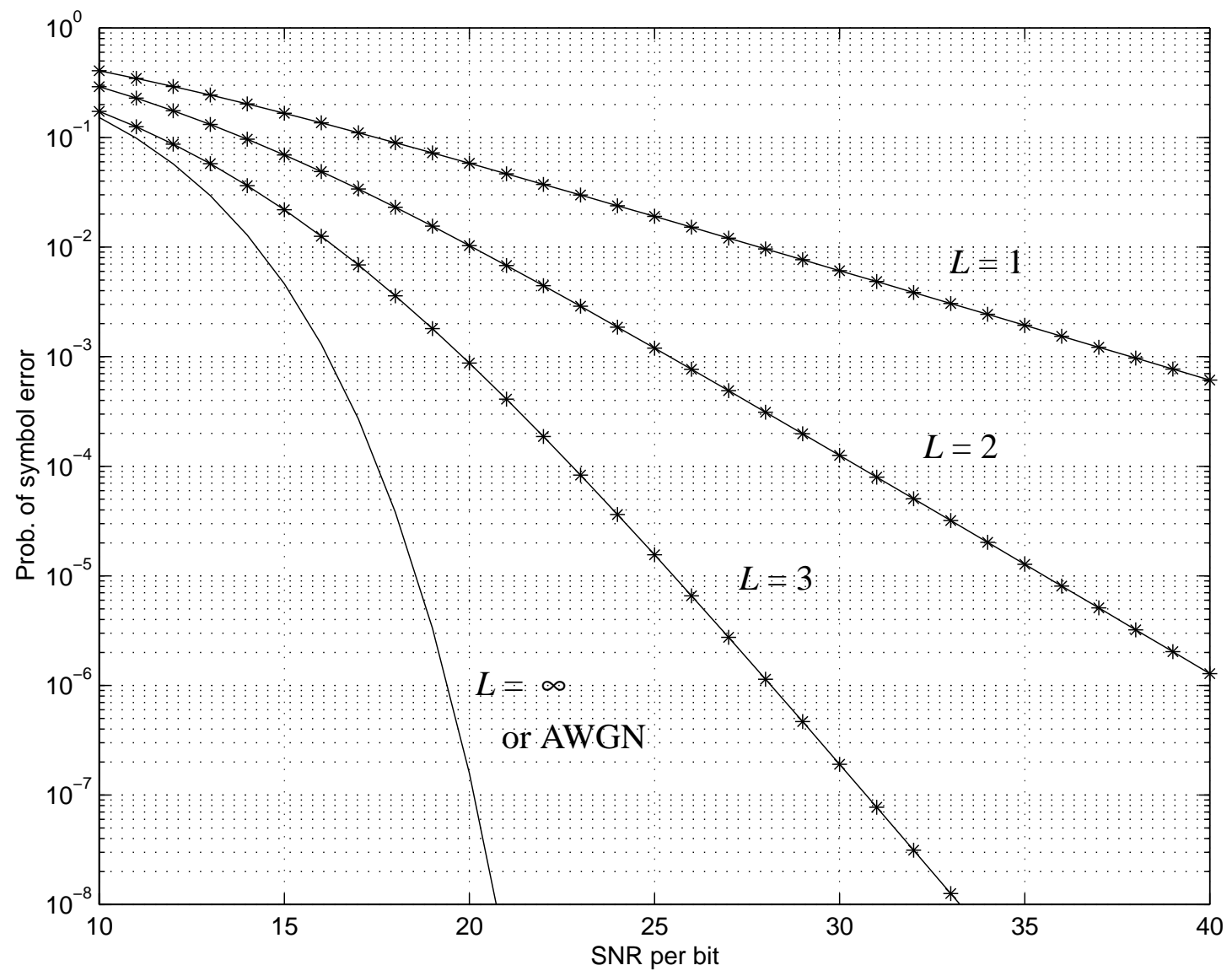

Figure -6 Matched filter bound SEP for 64-QAM transmission over $L$-flat fading diversity channels. 


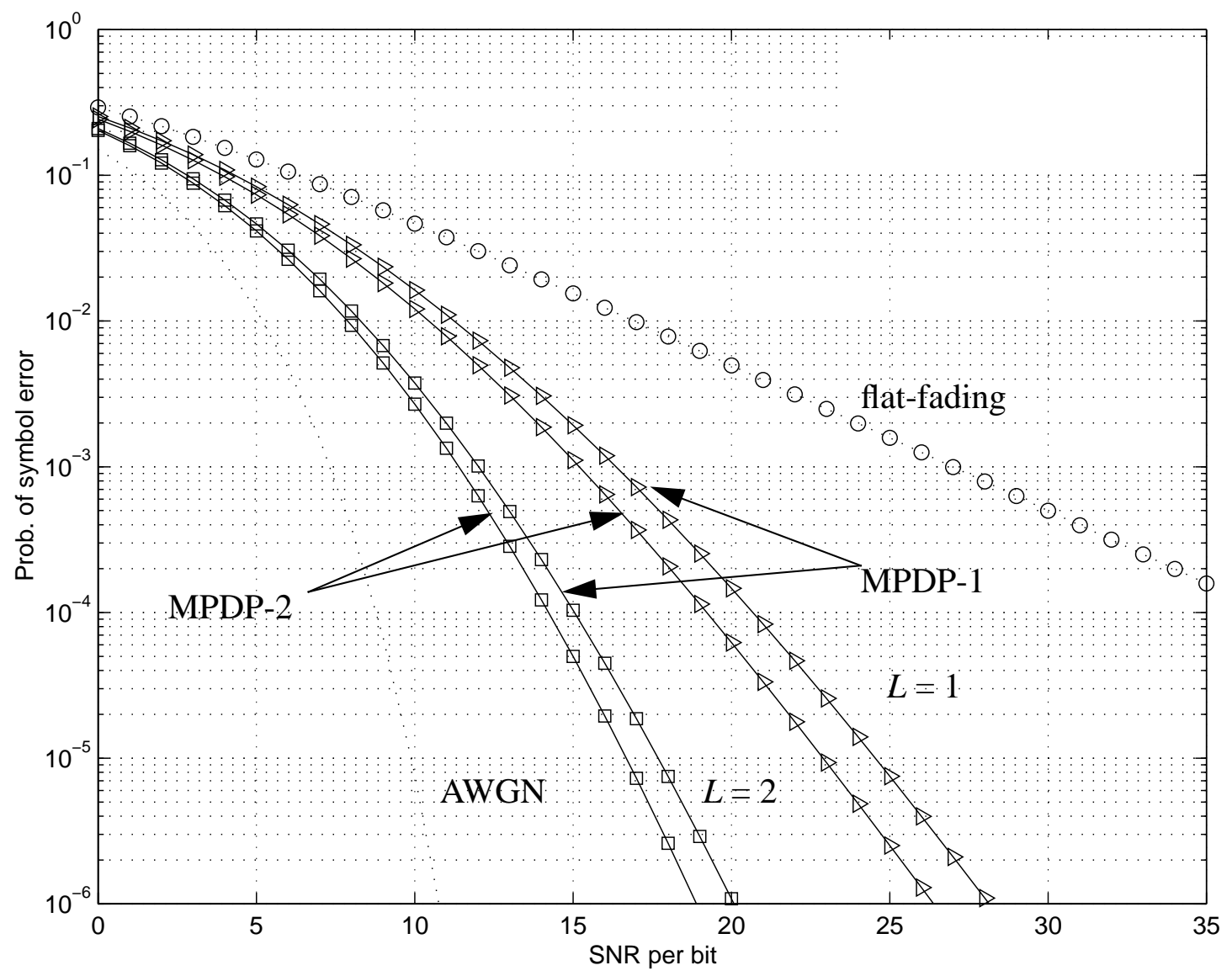

Figure -7 Matched filter bounds SEP for 4-QAM, $L=1,2$ 


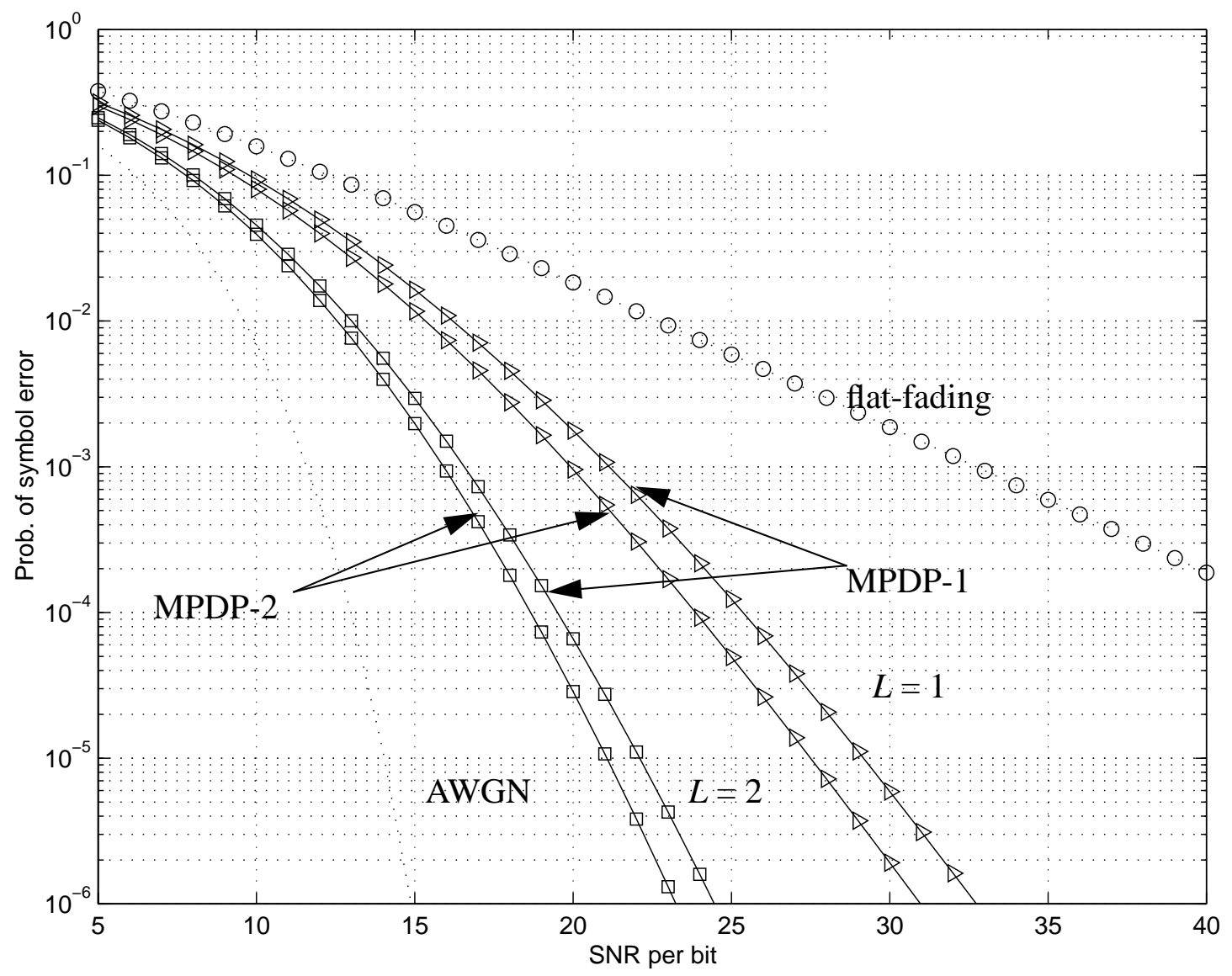

Figure -8 Matched filter bounds SEP for 16-QAM, $L=1,2$ 


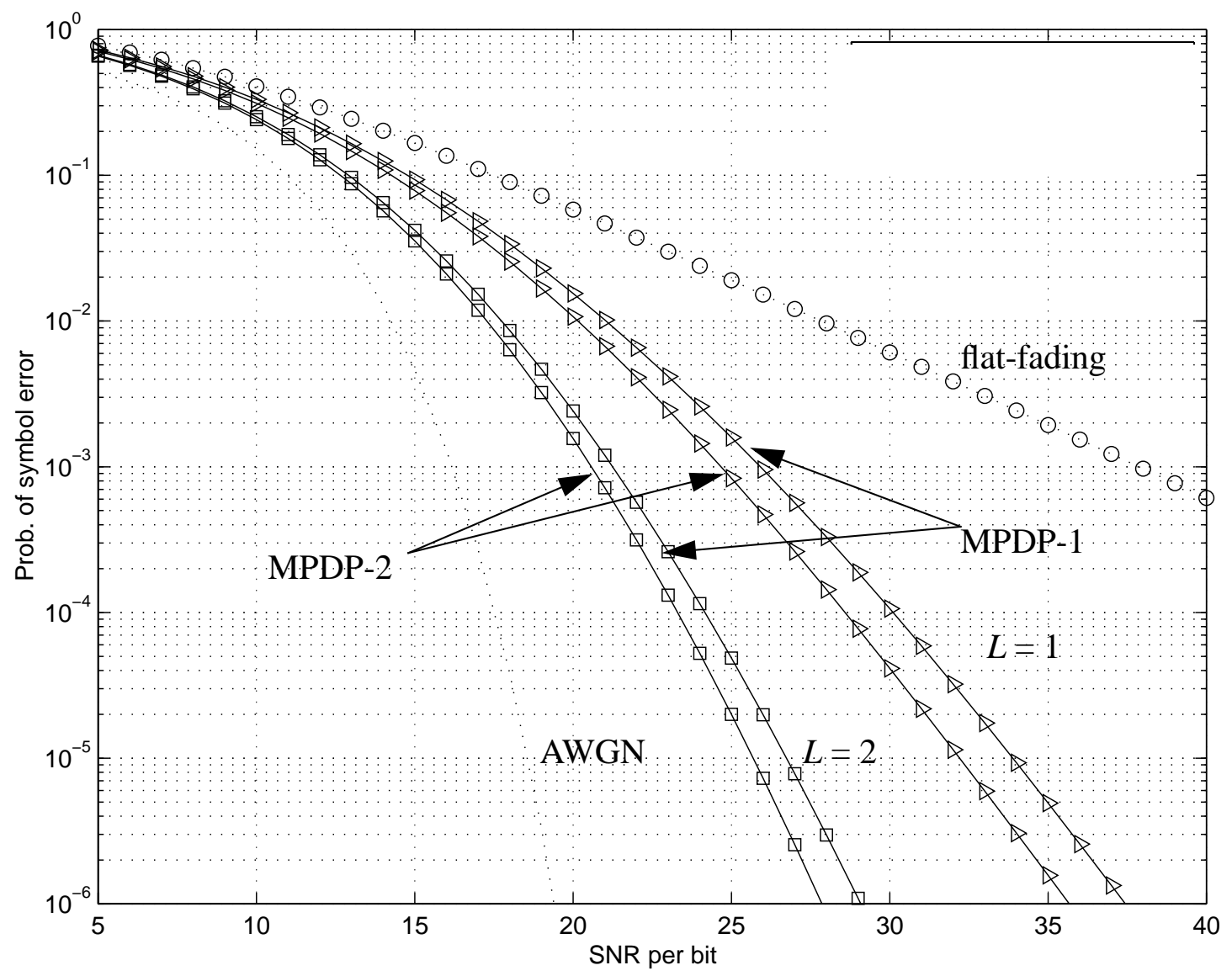

Figure -9 Matched filter bounds SEP for 64-QAM, $L=1,2$ 